\title{
A simple method for repairing membrane sinus perforation
}

\author{
Massimo Robiony $^{1}$, Giulia Tenani ${ }^{2}$, Massimo Sbuelz ${ }^{3}$, Matteo Casadei ${ }^{3}$ \\ ${ }^{1}$ Department of Maxillofacial Surgery, University-Hospital “S. Maria della Misericordia” of Udine, Udine, Italy \\ ${ }^{2}$ Private Practice, Udine, Italy \\ ${ }^{3}$ Department of Surgery, Section of Oral and Maxillofacial Surgery, University of Verona, Verona, Italy \\ Email: massimo.robiony@libero.it
}

Received 19 August 2012; revised 23 September 2012; accepted 27 September 2012

\begin{abstract}
The classical sinus lift procedure is a well-documented technique and Schneiderian membrane perforation is one of the most common complications. Various repairs have been developed for managing sinus membrane perforations. We describe a new suture technique of Schneiderian membrane that makes it possible to complete a sinus lift procedure that otherwise might have been aborted by preventing loss of the graft in the sinus cavity and secondary complications, such as sinusitis.
\end{abstract}

Keywords: Sinus; Lift; Membrane; Perforation

\section{INTRODUCTION}

The sinus lift procedure provides internal augmentation of the maxillary sinus with the goal of increasing the vertical bony dimension of the lateral maxilla to facilitate the use of dental implants to support an upper denture in patients with a severely atrophic maxilla. The classical sinus lift procedure, first introduced by Tatum [1] and Boyne and James [2], consists of preparing a top hinge door in the lateral maxillary sinus wall. The door is luxated inward and upward together with the Schneiderian membrane into a horizontal position, forming the new sinus base. The space underneath the lifted door and mucosa is filled with graft material. Although the sinus lift procedure is relatively safe, it does pose some potential problems. Maxillary sinus membrane perforation is the most common complication in sinus elevation augmentation surgery [3]. The Schneiderian membrane constitutes an important barrier for the defense of the sinus cavity. Its integrity is essential to maintaining the health and normal function of the sinus [4]. As reported in the literature [5-7], a perforation can represent a contraindication to continuing the surgery, because it can cause loss of the graft into the sinus, graft infection, early failure of the sinus lift, and sinusitis. In most instances, the perforation must be repaired so that it can hold particulate graft material and to complete the procedure [8]. Various techniques and materials have been developed for repairing perforated sinus membranes [9-16]. Here, we present a simple technique to manage a particular type of Schneiderian membrane perforation. This technique has been used with success on fifteen patients treated at the Clinic of Maxillofacial Surgery, Hospital S. Maria della Misericordia, Udine [17].

\section{CASE REPORT}

A 50-year-old male first visited the Maxillo-Facial Surgery Department of Azienda Ospedaliero-Uni-versitaria of Udine, Italy, in May 2010. He had severe maxillary and mandibular periodontitis that required total superior maxilla clearance and partial mandibular clearance of compromised dental elements. Orthopantomography (OPT) and computed tomography (CT) of the superior maxilla after 4 months revealed severe bone atrophy (Figure 1). We decided to perform a bilateral sinus lift procedure to facilitate implant positioning to support an upper denture. During the surgical procedure under general anesthesia, after elevating the sinus membrane, a small laceration of the sinus membrane occurred on the left side (Figure 2). This perforation was close to the inferior part of the top hinge door at the osteotomy site (Class I-II sec. Valassis-Fugazzotto) [13]. We decided to try to repair it by making two small holes in the bony door (Figure 3) and suturing the perforated Schneiderian membrane through them with a 4/0 Vicryl suture (Figures 4-5). Then, we sealed it with a resorbable collagen membrane, achieving continuity of the sinus membrane with this simple procedure. Finally, we filled the space underneath the lifted door and mucosa with graft material (Bio-Oss) (Figure 6) and covered it with additional resorbable collagen membrane before closing the oral mucosa, to complete the sinus lift operation. After another 4 months, the maxillary CT (Figure 7) exhibited new bone formation and an adequate amount of residual 

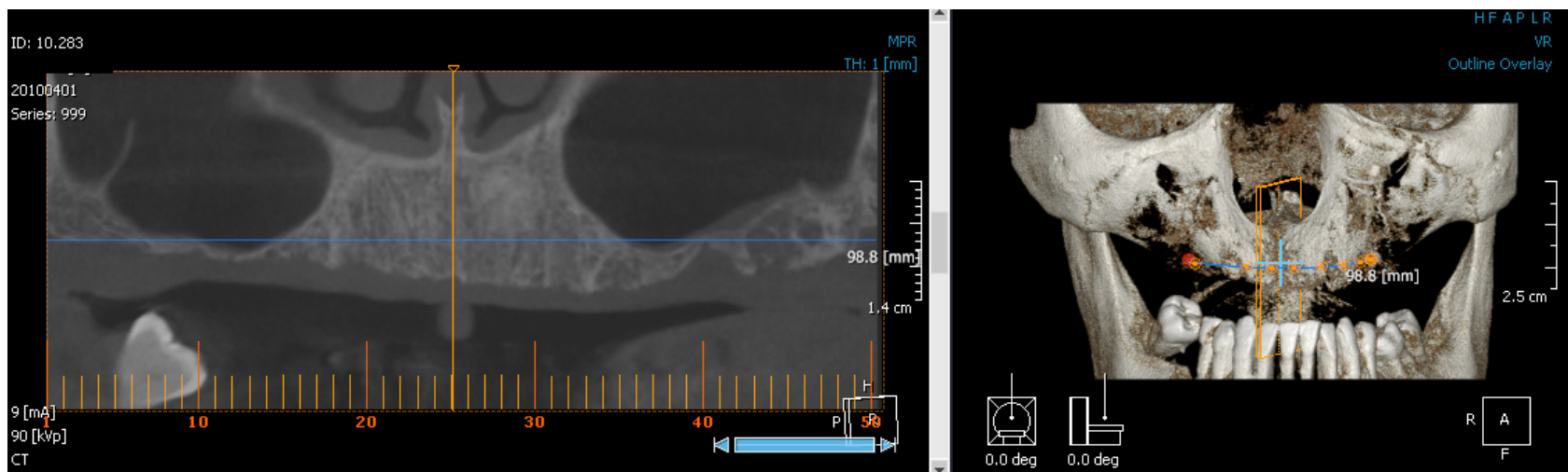

Figure 1. Preoperative CT, severe bone atrophy.

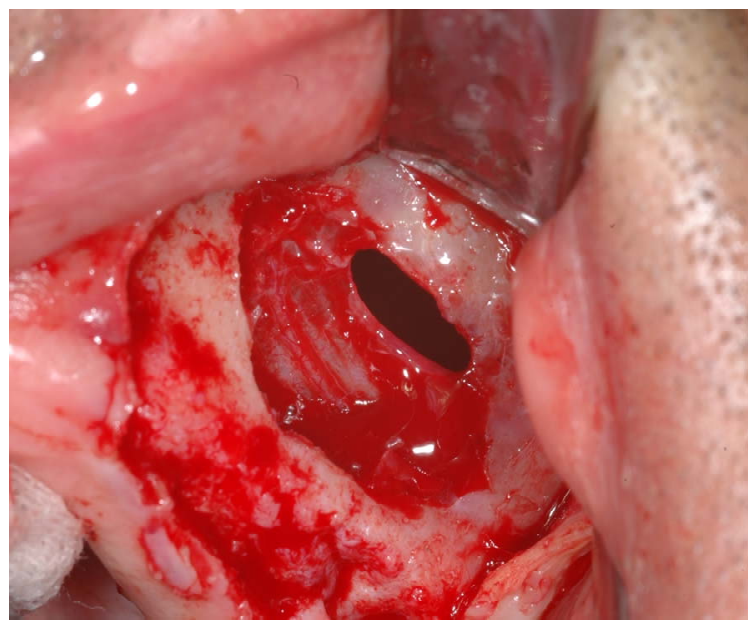

Figure 2. Intraoperative view 1, perforation of the membrane.

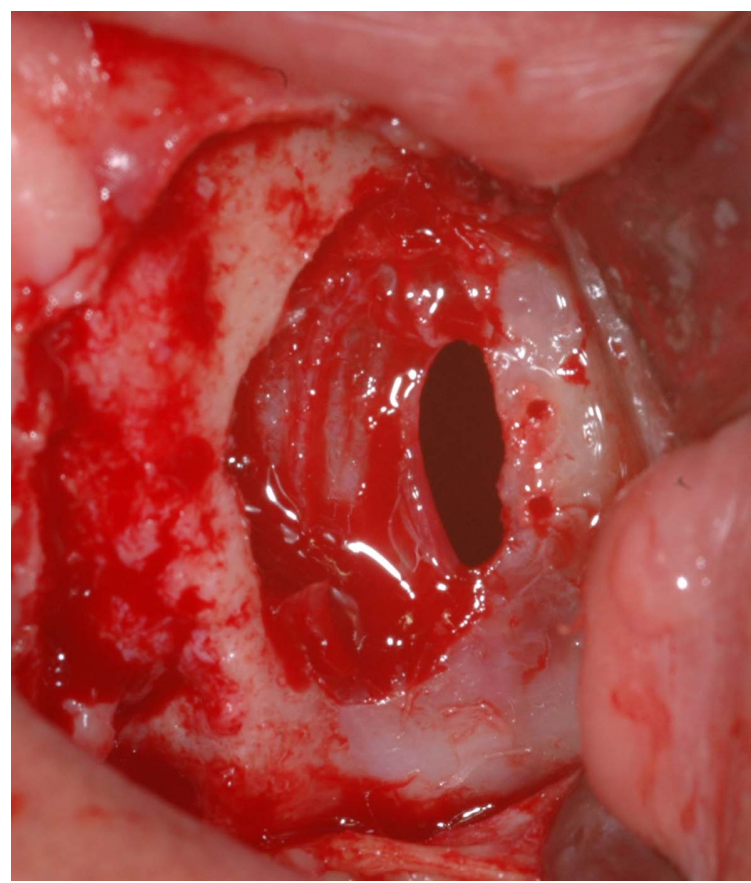

Figure 3. Intraoperative view 2, holes in the bony door.

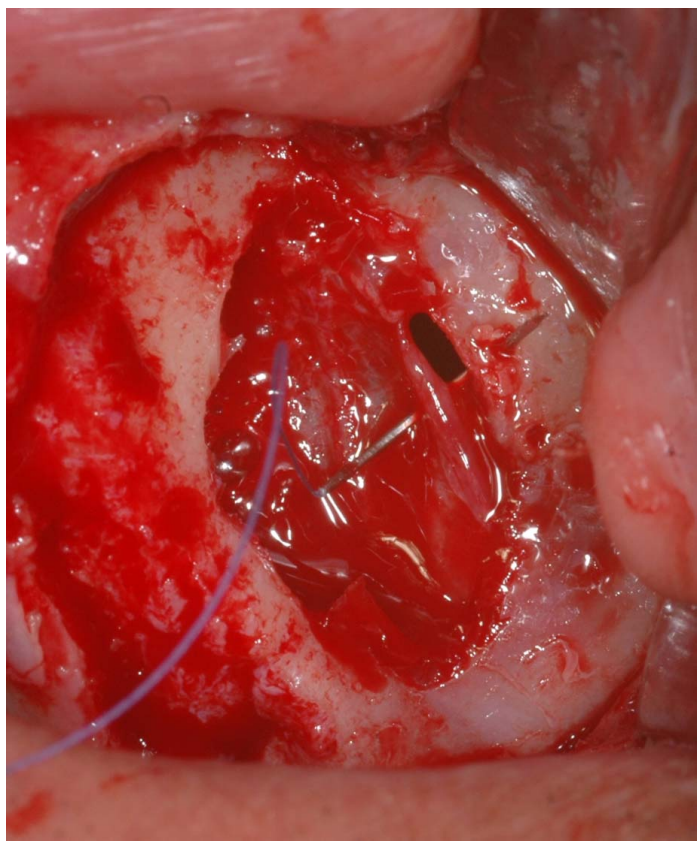

Figure 4. Intraoperative view 3 , suturing the perforated membrane through holes.

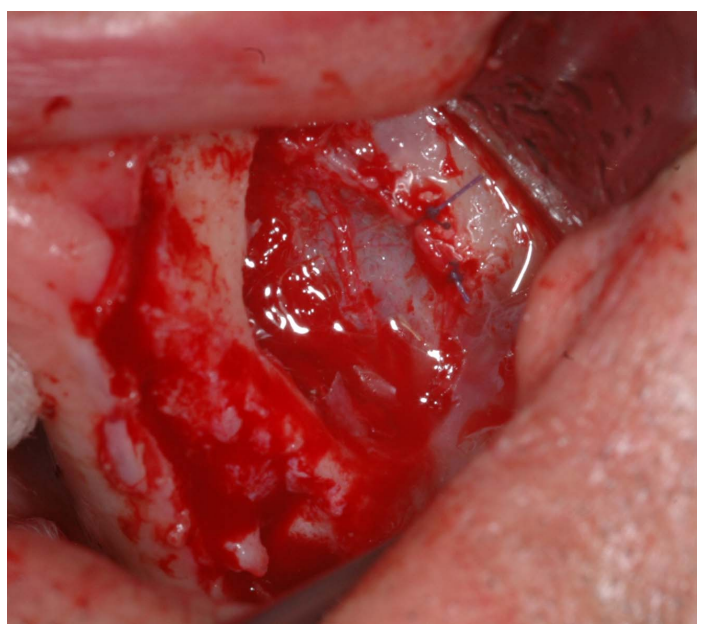

Figure 5. Intraoperative view 4, membrane sutured to the bone. 
graft material in both sites, with no significant differences between the right (no perforation of the Schneiderian membrane) and left (where the sinus membrane perforation occurred) sides. This allowed us to place three bone implants bilaterally. After positioning the implants, OPT was performed (Figure 8).

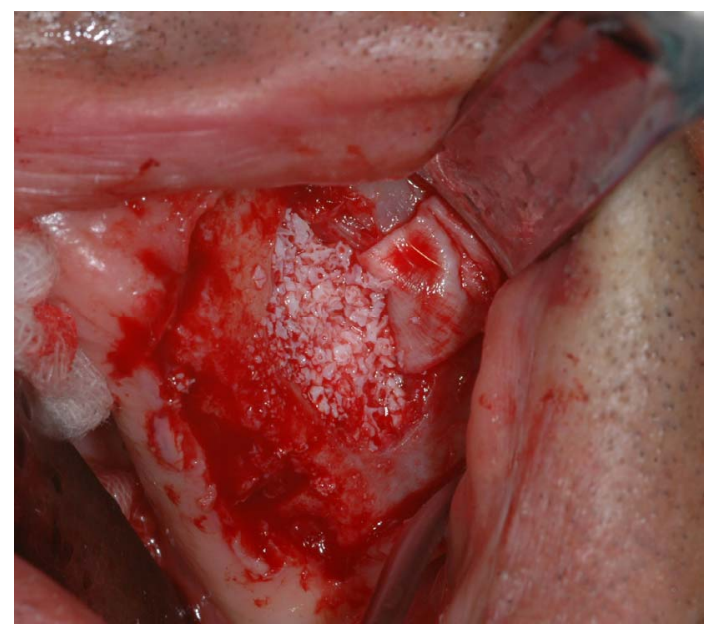

Figure 6. Intraoperative view 5, additional collagen membrane and graft material.

\section{DISCUSSION AND CONCLUSION}

The sinus lift procedure is a reliable operation that is not normally associated with a high incidence of major complications $[18,19]$. Perforation of the Schneiderian membrane is the most common complication of sinus elevation augmentation surgery [3]. There is no need for further intervention when the perforation is small and located in an area where the elevated mucosa folds together when lifting the door. If the perforation is larger and located in an unfavorable area, it needs to be closed and covered to prevent loss of the graft and secondary complications such as sinusitis [2,20]. Based on our experience of 15 patients treated as case described above, all successful, we propose suturing the membrane to the bony hinge door and using a resorbable collagen membrane to repair a specific type of sinus membrane perforation during sinus augmentation surgery. Although many authors did not recommend suturing of the membrane due to the extremely delicate features of the sinus membrane, where passing a suture will nearly always enlarge the existing perforation or create a new one [21], we argue that, when conditions permit, that is when the membrane is not too thin, this simple technique is an

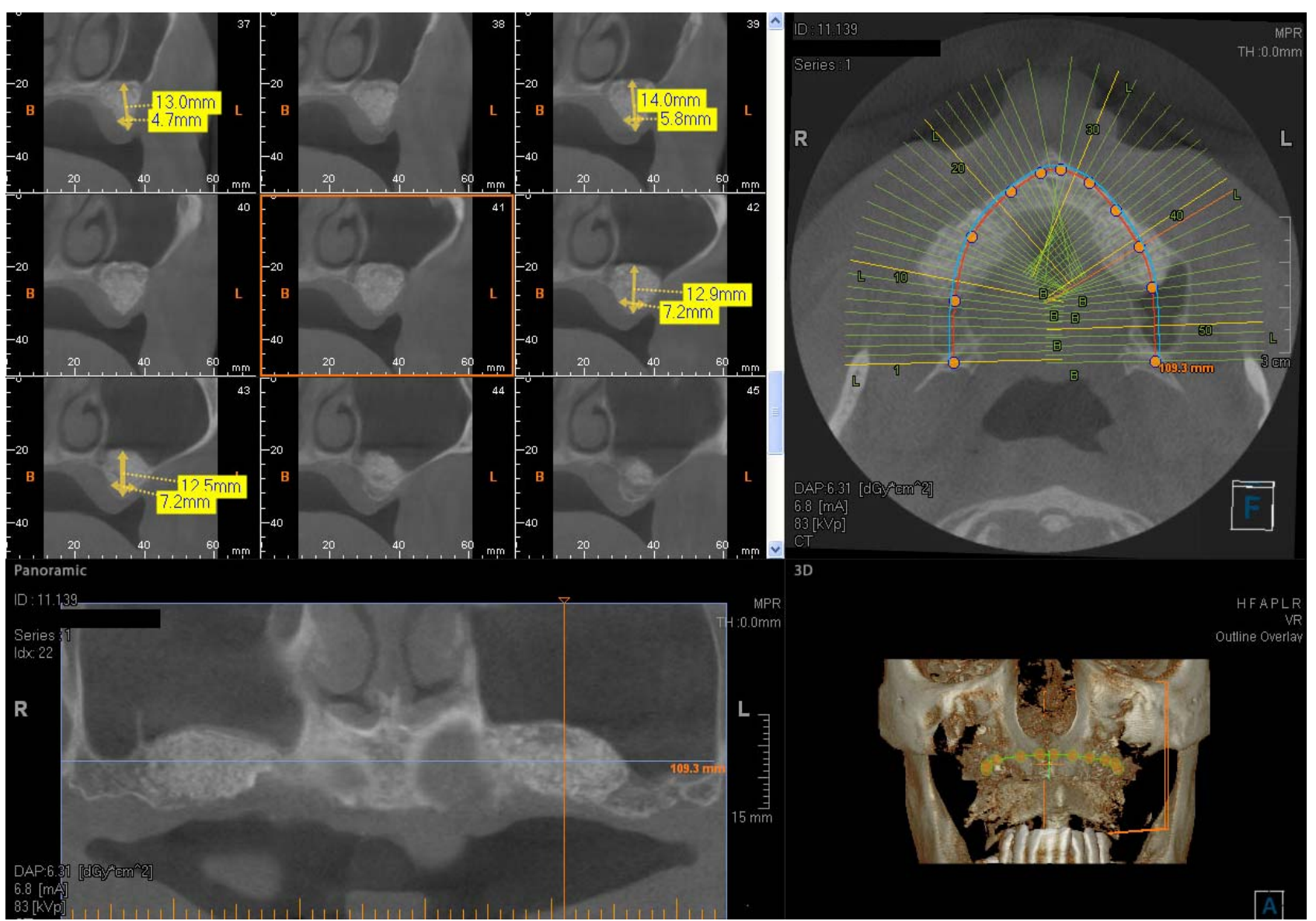

Figure 7. Postoperative CT, new bone formation and adequate amount of residual graft material. 


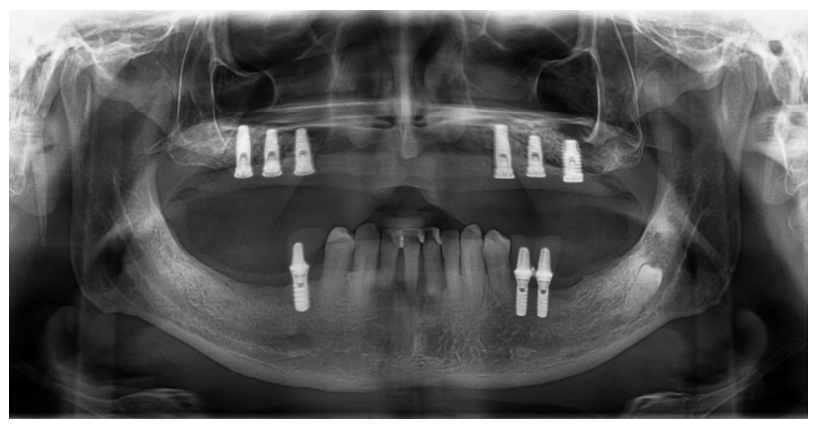

Figure 8. Post implants positioning OPT.

easy way to eliminate the membrane perforation, allowing the surgeon to continue the operation with a small risk of causing a second perforation and considerably reducing the incidence of secondary complications. In fact, this technique is the only one to ensure proper adhesion of the membrane to the inner bone surface of the maxillary sinus.

\section{REFERENCES}

[1] Tatum, O.H. (1986) Maxillary and sinus implant reconstruction. Dental Clinics of North America, 30, 207-229.

[2] Boyne, P. and James, R.A. (1980) Grafting of the maxillary sinus floor with autogenous marrow and bone. Journal of Oral and Maxillofacial Surgery, 17, 113-116.

[3] Schwartz-Arad, D., Herzberg, R. and Dolev, E. (2004) The prevalence of surgical complications of the sinus graft procedure and their impact on implant survival. Journal of Periodontology, 75, 511-516. doi:10.1902/jop.2004.75.4.511

[4] Ardekian, L., Oved-Peleg, E., Mactei, E.E. and Peled, M. (2006) The clinical significance of sinus membrane perforation during augmentation of the maxillary sinus. Journal of Oral and Maxillofacial Surgery, 64, 277-282. doi:10.1016/j.joms.2005.10.031

[5] Aimetti, M., Romagnoli, R., Ricci, G. and Massei, G. (2001) Maxillary sinus elevation: The effect of macrolacerations and microlacerations of the sinus membrane as determined by endoscopy. International Journal of Periodontics \& Restorative Dentistry, 21, 581-589.

[6] Konter, U. and Paper, H.D. (1995) Oroantral fistula and maxillary sinusitis after the sinus lift. Deutsche Zeitschrift für Mund-, Kiefer- und Gesichts-Chirurgie, 19, 32-34.

[7] Galli, S.K.D., Lebowitz, R.A., Giacchi, R.J., et al. (2001) Chronic sinusitis complicating sinus lift surgery. American Journal of Rhinology, 15, 181-186. doi:10.2500/105065801779954120

[8] Pjetursson, B.E., Tan, C.W., Zwahlen, M. and Lang, N.P. (2008) A systematic review of the success of sinus floor elevation and survival of implants inserted in combination with sinus floor elevation Part I: Lateral approach. Journal of Clinical Periodontology, 35, 216-240. doi:10.1111/j.1600-051X.2008.01272.x

[9] Proussaefs, P., Lozada, J. and Kim, J. (2003) Effects of sealing the perforated sinus membrane with a resorbable collagen membrane: A pilot study in humans. Journal of Oral Implantology, 29, 235-241.

doi:10.1563/1548-1336(2003)029<0235:EOSTPS >2.3.C $\underline{\mathrm{O} ; 2}$

[10] Proussaefs, P., Lozada, J., Kim, J. and Rohrer, M.D. (2004) Repair of the perforated sinus membrane with a resorbable collagen membrane: A human study. International Journal of Oral \& Maxillofacial Implants, 19, 413420.

[11] Pikos, M.A. (1999) Maxillary sinus membrane repair: Report of a technique for large perforations. Implant Dentistry, 8, 29-34. doi:10.1097/00008505-199901000-00003

[12] Pikos, M.A. (2008) Maxillary sinus membrane repair: Update on technique for large and complete perforations. Implant Dentistry, 17, 24-31. doi:10.1097/ID.0b013e318166d934

[13] Vallassis, J.M. and Fugazzotto, P.A. (1999) A classification system for sinus membrane perforations during augmentation with option to repair. Journal of Periodontology, 70, 692. doi:10.1902/jop.1999.70.6.692

[14] Testori, T., Wallace, S.S., Del Fabbro, M., Taschieri, S., Trisi, P., Capelli, M. and Weinstein, R.L. (2008) Repair of large sinus membrane perforations using stabilized collagen barrier membranes: surgical techniques with histologic and radiographic evidence of success. International Journal of Periodontics \& Restorative Dentistry, 28, 9-17.

[15] Kim, J.K., Hwang, J.W. and Yun, P.Y. (2008) Closure of large perforation of sinus membrane using pedicled buccal fat pad graft: A case report. International Journal of Oral \& Maxillofacial Implants, 23, 1139-1142.

[16] Hassani, A., Khojasteh, A. and Alikhasi, M. (2008) Repair of the perforated sinus membrane with buccal fat pad during sinus augmentation. Journal of Oral Implantology, 34, 330-333. doi:10.1563/1548-1336-34.6.330

[17] Politi, M., Robiony, M., Polini, F. and Costa, F. (2003) Bone biological box (BBB): An evolution of the sinus graft. Journal of Oral and Maxillofacial Surgery, 61, 1108-1112. doi:10.1016/S0278-2391(03)00327-6

[18] Mercier, P., Huang, H., Cholewa, J., et al. (1992) A comparative study of the efficacy and morbidity of five techniques for ridge augmentation of the mandible. Journal of Oral and Maxillofacial Surgery, 50, 210-217. doi:10.1016/0278-2391(92)90314-P

[19] Papa, F., Cortese, A., Maltarello, M.C., Sagliocco, R., Felice, P. and Claudio, P.P. (2005) Outcome of 50 consecutive sinus lift operations. British Journal of Oral and Maxillofacial Surgery, 43, 309-313. doi:10.1016/j.bjoms.2004.08.027

[20] Van Den Bergh, J.P.A., Ten Bruggenkate C.M., Disch F.J.M. and Tuinzing, D.B. (2000) Anatomical aspects of sinus floor elevations. Clinical Oral Implants Research, 11, 256-265. doi:10.1034/j.1600-0501.2000.011003256.x

[21] Vlassis, J.M. and Fugazzotto, P.A. (1999) A classification system for sinus membrane perforations during augmentation procedures with options for repair. Journal of $\mathrm{Pe}$ riodontology, 70, 692-699. doi:10.1902/jop.1999.70.6.692 